\title{
Plasmon-resonant Raman spectroscopy in metallic nanoparticles: Surface-enhanced scattering by electronic excitations
}

\author{
R. Carles, ${ }^{*}$ M. Bayle, P. Benzo, G. Benassayag, and C. Bonafos \\ CEMES-CNRS-Université de Toulouse, rue Jeanne Marvig, BP 94347, 31055 Toulouse Cedex 4, France \\ G. Cacciato ${ }^{\dagger}$ and V. Privitera \\ CNR-IMM, via S. Sofia 64, 95123 Catania, Italy \\ (Received 20 July 2015; revised manuscript received 12 October 2015; published 5 November 2015)
}

\begin{abstract}
Since the discovery of surface-enhanced Raman scattering (SERS) 40 years ago, the origin of the "background" that is systematically observed in SERS spectra has remained questionable. To deeply analyze this phenomenon, plasmon-resonant Raman scattering was recorded under specific experimental conditions on a panel of composite multilayer samples containing noble metal ( $\mathrm{Ag}$ and $\mathrm{Au}$ ) nanoparticles. Stokes, anti-Stokes, and wide, including very low, frequency ranges have been explored. The effects of temperature, size (in the nm range), embedding medium $\left(\mathrm{SiO}_{2}, \mathrm{Si}_{3} \mathrm{~N}_{4}\right.$, or $\left.\mathrm{TiO}_{2}\right)$ or ligands have been successively analyzed. Both lattice (Lamb modes and bulk phonons) and electron (plasmon mode and electron-hole excitations) dynamics have been investigated. This work confirms that in Ag-based nanoplasmonics composite layers, only Raman scattering by single-particle electronic excitations accounts for the background. This latter appears as an intrinsic phenomenon independently of the presence of molecules on the metallic surface. Its spectral shape is well described by revisiting a model developed in the 1990s for analyzing electron scattering in dirty metals, and used later in superconductors. The $g_{s}$ factor, that determines the effective mean-free path of free carriers, is evaluated, $g_{s}^{\text {expt }}=0.33 \pm 0.04$, in good agreement with a recent evaluation based on time-dependent local density approximation $g_{s}^{\text {theor }}=0.32$. Confinement and interface roughness effects at the nanometer range thus appear crucial to understand and control SERS enhancement and more generally plasmon-enhanced processes on metallic surfaces.
\end{abstract}

DOI: 10.1103/PhysRevB.92.174302

\section{INTRODUCTION}

Surface-enhanced Raman scattering (SERS) was discovered 40 years ago on pyridine adsorbed on a rough silver electrode [1]. The impressive enhancement, up to $10^{8}$, of the vibrational fingerprint of molecules located at specific sites ("hot spots") on the surface of metallic particles is responsible of a further huge development of Raman spectrometry [2]. The possibilities of acquiring vibrational information down to a single molecule $[3,4]$ and controlling a single hot spot with high spatial resolution using tip-enhanced Raman spectroscopy (TERS) [5-8] have led to new insights on local mechanisms at the origin of SERS. From the beginning, these mechanisms have been commonly referred to as electromagnetic (EM) [9] when due to local electromagnetic inductive effects, or chemical (CM) [10] when due to resonant charge transfer effects, the first one giving the largest contribution to the enhancement factor [11].

Although the development of many theoretical refinements and the fact that SERS has become a widespread analytical technique (i.e., the number of associated publications overcomes $2 \times 10^{4}$ ), a lot of important questions remain to be clearly solved [12]. Among them, the presence of a continuous signal, the so-called "background," on which the molecular signatures are superimposed in all SERS spectra, is still subject to peculiar attention. Since the pioneering works on this subject [13-16], the background generated by rough silver surfaces

\footnotetext{
*robert.carles@cemes.fr

${ }^{\dagger}$ Also at: Dipartimento di Fisica ed Astronomia-Università di Catania, via S. Sofia 64, 95123 Catania, Italy.
}

PACS number(s): 74.25.nd, 63.22.Kn, 73.22.-f, 78.30.Er or nude silver nanoparticles has been undoubtedly ascribed to Raman scattering by electron-hole excitations. It has been observed that the shape of this signal is related to the size of the metal particles [16], and a linear dependence of the frequency of its maximum intensity versus the inverse of the size of silver nanocrystals (Ag-NCs) has been demonstrated [17]. More recently, the background dependence on local morphology and surface roughness has been once more underlined [18], and a direct link between the line shape of the background and the mean diameter of the NCs assembly has been put forward using a phenomenological approach $[19,20]$.

For SERS on molecules grafted on gold nanoparticles, the background is presented as resulting from either luminescent processes [21] or electronic Raman scattering [22]. This background also appears in TERS measurements and its origin is here also subject to various interpretations, such as far-field contribution, luminescence from the tip itself, or molecular fluorescence $[6,23,24]$. The possible role of luminescence processes, mainly with gold- (or copper-) based nanostructures, cannot be discarded because of the presence of real electronic (interband) transitions in the visible range [25]. Moreover, a strong dependence on this background signal on the presence of molecules that have their own electronic response and excitations has been also underlined [10,26,27].

Analyzing more precisely the spectral shape of the background is thus still of high interest, either experimentally or theoretically, because this signal does appear as an intrinsic phenomenon that cannot be separated from the SERS effect $[18,19,28,29]$. The link between SERS and background signal is evidenced by the same resonant behavior versus excitation wavelength $[20,29]$, the same dependence on tip-sample distance in TERS experiments [5], and same time dependence 
for the blinking phenomenon [27]. Until now, no model has been proposed to quantitatively analyze the whole spectral shape of the background and in particular its correlation with the surface geometry. This point is critical for detailed diagnostics and improvement in the efficiency of devices based on SERS.

This work deals with experimental analysis of inelastic scattering by both electronic and vibrational excitations in metallic nanocrystals ( $M$-NCs, with $M=\mathrm{Ag}$ or $\mathrm{Au}$ ) embedded in a dielectric matrix. Various embedding media are successively used $\left(\mathrm{SiO}_{2}, \mathrm{Si}_{3} \mathrm{~N}_{4}\right.$, or $\mathrm{TiO}_{2}$ and molecular ligands). Specific samples have been elaborated to take simultaneously benefit of bilayer interference and plasmon-resonance enhancements. In the first part, we mainly analyze the intrinsic generation of a background on Ag nanostructures without the presence of molecules. We particularly focus on the response of free electronic excitations recording Stokes and anti-Stokes Raman spectra over a wide frequency range including the very lowfrequency range. The effects of temperature and confinement in nm-sized NCs are successively analyzed. It is confirmed that the so-called background is present even in the absence of molecules on the metal surface and that it concerns both Stokes and anti-Stokes processes. The line shape of the electronic inelastic scattering from Ag-NCs assemblies and its dependence on confinement are quantitatively accounted for adapting a model already used in dirty metals [30], superconductors [31,32], or highly doped semiconductors [33].

\section{EXPERIMENT AND METHODS}

\section{A. Sample design}

To counterbalance the Raman signal weakness coming from a very small volume of matter, specific substrates have been used. They consist of a transparent dielectric layer (refractive index $n$ ) deposited (or grown) on a Si substrate. The layer thickness $t$ is adapted to tune with one of the wavelengths $\lambda_{m}$ ensuring antireflectance condition to the localized surface plasmon-polariton resonance (LSPR) of the metallic particles under investigation, $\lambda_{m}=\lambda_{\mathrm{LSPR}}$, with

$$
t=\left(m+\frac{1}{2}\right) \frac{\lambda_{m}}{2 n},
$$

$\lambda_{m}$ being the electromagnetic wavelength in vacuum and $m$ the destructive interference order. When the excitation laser wavelength $\lambda_{i}$ is chosen near this coincidence, $\lambda_{i} \simeq \lambda_{\mathrm{LSPR}}$, one can simultaneously take benefit of both optical amplification and plasmon-resonant enhancement [19,34]. In this work, several embedding media $\left(\mathrm{SiO}_{2}, \mathrm{Si}_{3} \mathrm{~N}_{4}, \mathrm{TiO}_{2}\right.$, or ligands in air) and two noble metals ( $\mathrm{Ag}$ or $\mathrm{Au}$ ) have been used. The average size of the $M$-NCs is in the nm range and thus the dipolar approximation holds. The extinction efficiency $C_{e x}$ of a nm-sized metallic sphere (diameter $D$ ) embedded in a transparent medium (dielectric function $\varepsilon_{m}$ ) is essentially due to absorption and one gets

$$
C_{e x} \simeq C_{\mathrm{abs}}=12 \pi \frac{D}{\lambda} \varepsilon_{m}^{3 / 2} \frac{\varepsilon^{\prime \prime}(\lambda)}{\left\{\left[\varepsilon^{\prime}(\lambda)+2 \varepsilon_{m}\right]^{2}+\left[\varepsilon^{\prime \prime}(\lambda)\right]^{2}\right\}},
$$

where $\varepsilon(\lambda)=\varepsilon^{\prime}+i \varepsilon^{\prime \prime}$ is the complex dielectric function of the metal. The relaxation of the plasmon (wavelength $\lambda_{p}$ in bulk)

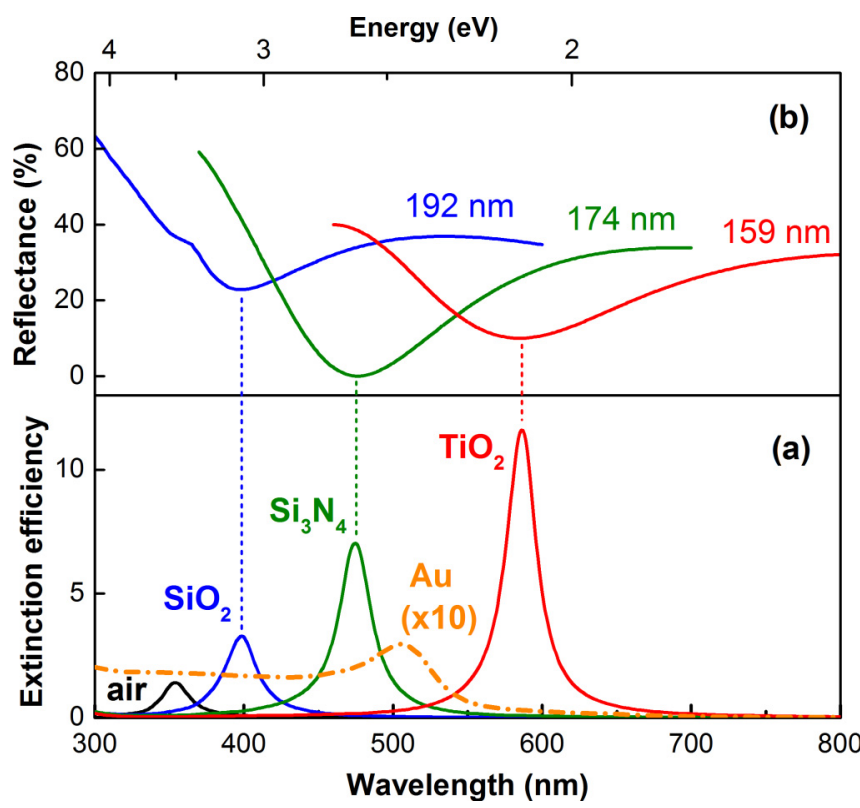

FIG. 1. (Color online) Theoretical simulation illustrating the tun-

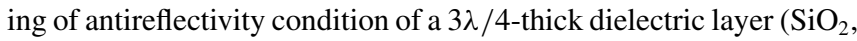
$\mathrm{Si}_{3} \mathrm{~N}_{4}$, or $\mathrm{TiO}_{2}$ ) on $\mathrm{Si}$ and LSPR in a spherical metallic nanoparticle of diameter $4 \mathrm{~nm}$ : (a) extinction efficiency of an embedded Ag particle, (b) reflectance of the heterostructure. In (a) is also plotted in the dashed-dotted line the extinction efficiency of a gold nanoparticle covered by a dielectric of index 1.33 (note the amplification factor of 10)

due to finite-size effect has been taken into account by simply adding (in the 1-10 $\mathrm{nm}$ range) a supplementary term at the imaginary part $\varepsilon^{\prime \prime}$ of the bulk dielectric function [35]

$$
\begin{gathered}
\varepsilon_{s}^{\prime \prime}=\frac{\lambda^{3}}{2 \pi c \lambda_{p}^{2} \tau_{s}} \\
\text { with } \quad \Gamma_{s}=\frac{\hbar}{\tau_{s}}=2 \hbar g_{s} \frac{v_{F}}{D} .
\end{gathered}
$$

In Eq. (4), $\Gamma_{s}$ is a damping energy parameter associated to a surface collision time $\tau_{s}$, and $v_{F}$ is the Fermi velocity. The geometrical coefficient $g_{s}$ allows to define an effective meanfree path for electron collisions with regard to the diameter $D$ [36]. In Fig. 1(a), which is here only schematic, we have adopted the most common value $g_{s}=1$ for calculating $C_{e x}$. The theoretical and experimental determinations of $g_{s}$ will be discussed in detail further.

One observes in Fig. 1(a) that the resonance quality factor, defined as $Q=\lambda_{\mathrm{LSPR}} / \Delta \lambda$ where $\Delta \lambda$ is the FWHM, is higher for Ag-NCs than for Au-NCs (metal effect), as expected, because of the supplementary damping by interband transitions in gold. Moreover, the higher the refractive index of the embedding matrix, the higher this quality factor (matrix effect), following the sequence air $(n=1), \mathrm{SiO}_{2}(1.46), \mathrm{Si}_{3} \mathrm{~N}_{4}(2.07)$, $\mathrm{TiO}_{2}$ (2.57). This enhancement of the dielectric confinement is accompanied by a red-shift of the LSPR.

The modeled reflectance spectra of the corresponding bilayers are reported in Fig. 1(b). The propagation of electromagnetic waves in the heterostructure was described using in a matrix formulation the continuity of the magnetic and electric 
TABLE I. Characteristics of the samples analyzed in this work. The thickness is that of the embedding matrix of the metallic nanocrystals. The kinetic energy and dose are those of the implanted $\mathrm{Ag}^{+}$ions. The average diameters $\bar{D}$ have been deduced by analyzing TEM plan views or using the Raman signature of the dominant Lamb mode, respectively. The number in the last column refers to the figure where the respective Raman spectra are reported.

\begin{tabular}{|c|c|c|c|c|c|c|c|c|}
\hline Sample & Metal & Matrix & Thickness (nm) & Energy $(\mathrm{keV})$ & Dose $\left(10^{15} / \mathrm{cm}^{2}\right)$ & $\bar{D}_{\mathrm{TEM}}(\mathrm{nm})$ & $\bar{D}_{\text {Raman }}(\mathrm{nm})$ & Figure \\
\hline A & $\mathrm{Ag}$ & $\mathrm{SiO}_{2}$ & 1000 & 190 & 40 & $6.0 \pm 1.5$ & $6.0 \pm 0.4$ & $2,4,7$ \\
\hline B & $\mathrm{Ag}$ & $\mathrm{SiO}_{2}$ & 240 & 3 & 4.7 & $4.6 \pm 1.0$ & $3.5 \pm 0.2$ & $3,5,8$ \\
\hline $\mathrm{C}$ & $\mathrm{Ag}$ & $\mathrm{SiO}_{2}$ & 240 & 3 & 7.1 & $5.8 \pm 1.0$ & $4.0 \pm 0.3$ & 8 \\
\hline D & $\mathrm{Ag}$ & $\mathrm{SiO}_{2}$ & 240 & 3 & 11.8 & $5.4 \pm 1.0$ & $4.8 \pm 0.3$ & 8 \\
\hline $\mathrm{E}$ & $\mathrm{Ag}$ & $\mathrm{Si}_{3} \mathrm{~N}_{4}$ & 160 & 8 & 18 & $2.8 \pm 0.7$ & $2.8 \pm 0.6$ & 9 \\
\hline $\mathrm{F}$ & $\mathrm{Ag}$ & $\mathrm{TiO}_{2}$ & 89 & 20 & 30 & $\simeq 3.0$ & $2.8 \pm 0.3$ & 10 \\
\hline G & $\mathrm{Au}$ & TDSP & 90 & & & $5.0 \pm 0.8$ & $5.0 \pm 0.2$ & 11 \\
\hline
\end{tabular}

displacement field components at the different interfaces $[19,34]$. The input data are the complex optical constants of the substrate $(\mathrm{Si})$ and dielectric layer (amorphous $\mathrm{SiO}_{2}$, amorphous $\mathrm{Si}_{3} \mathrm{~N}_{4}$, and polycrystalline rutile). In each case, the dielectric thickness has been chosen in order to realize a $3 \lambda / 4$ antireflective system $[m=1$ in Eq. (1)] at the LSPR wavelength $\lambda_{\text {LSPR }}$ of the embedded $M$-NCs $(M=\mathrm{Ag}$ or Au).

\section{B. Optical measurements: Original setup and procedure}

When the reflectance is minimum, the total electromagnetic field is maximum at the antinodes of the stationary wave into the dielectric. In particular, this maximum occurs at the free surface of the bilayer [19]. To enhance the interaction between electrons in the $M$-NCs and polaritons in the dielectric, it is thus particularly relevant to place these NCs at the near vicinity of this free surface $[19,20]$. As a consequence, two techniques have been used: either (a) low-energy implantation of $\mathrm{Ag}^{+}$ions leading to the formation of a single plane of $\mathrm{Ag}-\mathrm{NCs}$ embedded at few nanometer distance beneath the free surface [37], or (b) deposition on this surface of assemblies of colloidal Au-NCs. The characteristics of the samples are reported in Table I.

Raman spectra were recorded over a wide frequency range using a T64000 Horiba Jobin Yvon triple spectrometer. To record the inelastic scattered signal at very low frequency, the standard backscattering geometry is replaced by a twoobjective system (Fig. 2): the laser beam is focused on the sample by a first objective (12 $\mathrm{mm}$ focal length) under an oblique incidence, whereas the scattered light is collected in quasinormal incidence through a microscope objective (0.55 numerical aperture, $\times 40$ magnification, long-working distance). Moreover, a specific optical and electronic filter (labeled F in Fig. 2) is inserted into the spectrometer to limit the spectral window. One gets a very high level of rejection on elastically scattered photons into the spectrometer allowing as a consequence to record very low-frequency Raman-Brilouin spectra and the Rayleigh line. In Fig. 2, an example of Raman spectra recorded on embedded Ag-NCs in $\mathrm{SiO}_{2}$ (red dotted line) and on a zone without Ag-NCs (green continuous line) for sample $\mathrm{A}$, is presented. In the latter spectrum, one clearly sees that this specific system allows to record the Brillouin peak of the Si substrate near $7.1 \mathrm{~cm}^{-1}$. The standard backscattering geometry is used either for recording the Raman signal under a laser illumination for frequency shift higher than $30 \mathrm{~cm}^{-1}$ (dotted blue line in Fig. 2), or for recording the response of the system, with or without filtering, using a calibrated white lamp (black dotted line in Fig. 2).

The laser beam power was kept lower than $1 \mathrm{~mW}$ limiting thus heating effects. For each composite layer, a proper laser wavelength and a specific thickness of the dielctric layer were chosen in order to record Raman scattering spectra in plasmon-resonant conditions (see Table I). For temperature measurements, the sample was glued on the finger of a nitrogen-cooled microcryostat (Physics Instruments), allowing also heating up to $500 \mathrm{~K}$. Most of the presented spectra result from an average of tens of spectra recorded using an $\mathrm{X}-\mathrm{Y}$ piezoscanning. A spatial resolution around $1 \mu \mathrm{m}^{2}$ was obtained using a confocal pinhole.

The reflectance of the samples has been recorded in quasinormal incidence $\left(6^{\circ}\right)$ using a Varian Cary 5000 UV-visible spectrometer. An example of reflectance spectra recorded on

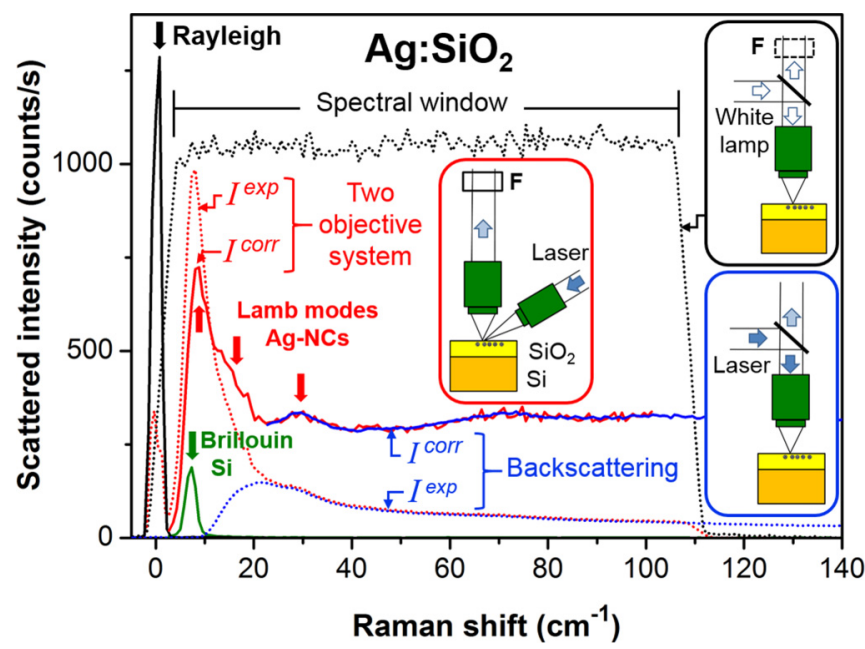

FIG. 2. (Color online) Different geometries and sample design adopted for recording and enhancing the Raman signal at very low frequency. The sample corresponds to embedded Ag-NCs in $\mathrm{SiO}_{2}$ (sample A). The spectral window can be limited by a specific optical and electronic filter $(\mathrm{F})$ inserted into the spectrometer, as shown by the response under white light illumination (black dotted line). Rough $\left(I^{\text {expt }}\right)$ and corrected $\left(I^{\text {corr }}\right)$ Raman spectra are reported in dotted and continuous lines, respectively. $I^{\text {corr }}$ is defined by Eq. (6) and is reported with an amplification factor of 20 . The Brillouin signal of the Si substrate (green continuous line) has been recorded from a zone of the sample without Ag-NCs. 


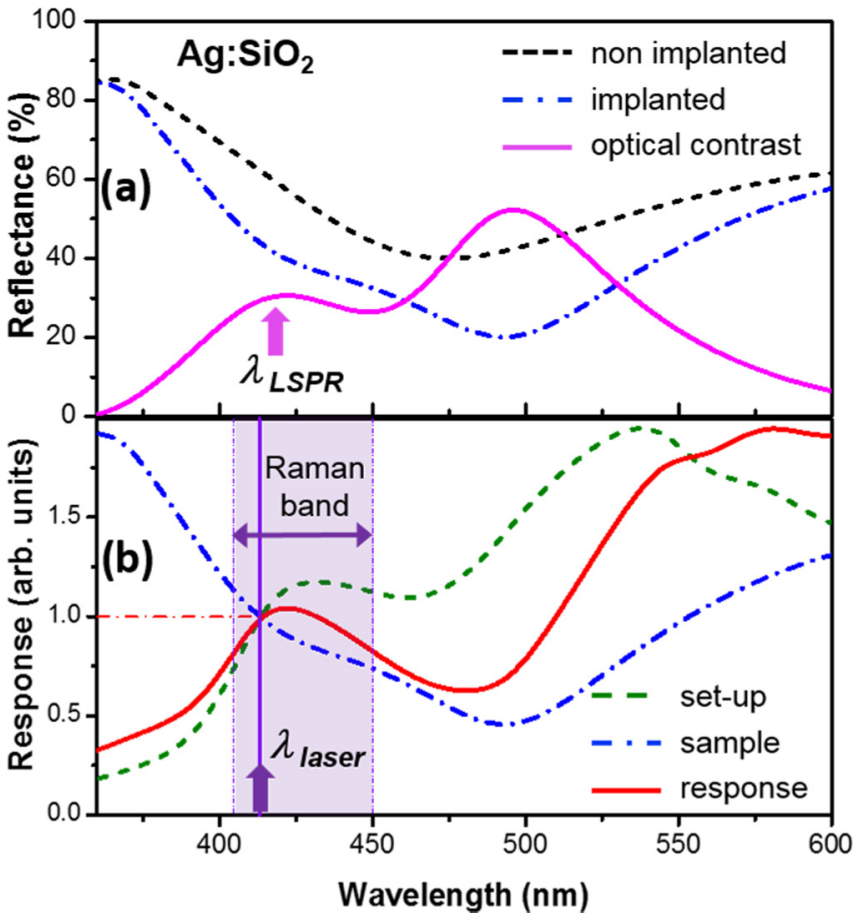

FIG. 3. (Color online) (a) Reflectance spectrum of sample B from a zone with (dotted-dashed blue line) or without (dashed black line) Ag-NCs. The optical contrast $C_{R}$ is reported in continuous line. (b) Reflectance spectrum of a zone with Ag-NCs embedded in $\mathrm{SiO}_{2}$ (dotted-dashed blue line), response of the spectrometer (dashed green line), and optical response of the whole experimental setup (continuous red line). These responses have been normalized to 1 at the laser wavelength $\left(\lambda_{i}=413 \mathrm{~nm}\right)$. The frequency range corresponding to a Raman spectrum spaning from $500 \mathrm{~cm}^{-1}$ in the anti-Stokes part to $2000 \mathrm{~cm}^{-1}$ in the Stokes part is indicated.

sample B is shown in Fig. 3(a). By comparing signals recorded from zone with $(R)$ and without $\left(R_{0}\right)$ Ag-NCs, one can define an optical contrast as

$$
C_{R}=1-\frac{R}{R_{0}}
$$

On the spectrum of $C_{R}$ [continuous curve in in Fig. 3(a)], one clearly observes the LSPR signature of Ag-NCs embedded in $\mathrm{SiO}_{2}$ around $420 \mathrm{~nm}$ in rather good agreement with the simulated absorption spectrum (Fig. 1). The second bump near $490 \mathrm{~nm}$ is attributed to the modulation of the $m=1$ reflectance minimum [Eq. (1)] induced by the insertion of Ag-NCs in the dielectric layer. By fitting the optical contrast curve with an appropriate modeling of the optical response of the heterostructure, it has been already shown that an average size of the Ag-NCs can be deduced [34]. In Fig. 3(b), the reflectance spectrum (blue dotted-dashed line) and the spectral response of the spectrometer (green dotted line) are reported. The latter has been determined using a calibrated white lamp and using the experimental geometry schematized in Fig. 2. The total response $f\left(\omega_{i}, \omega\right)$ of the setup (sample + spectrometer) has been determined using the procedure reported in Fig 2. An example (for sample B) is reported in Fig. 3(b), where the total setup response (continuous red curve) is deduced by multiplying the sample reflectance by

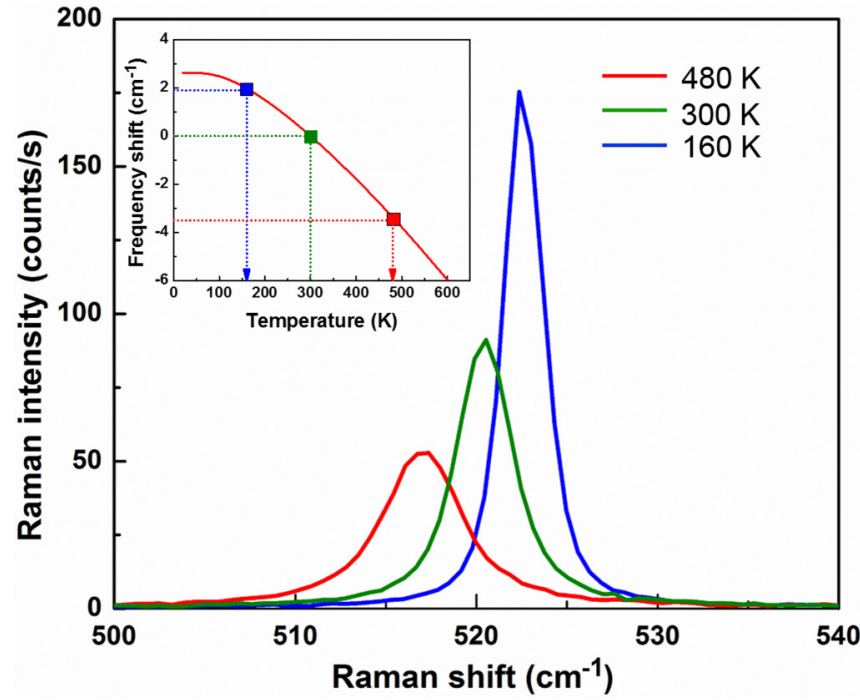

FIG. 4. (Color online) Detail of the rough Raman spectra of sample A with Ag-NCs embedded in $\mathrm{SiO}_{2}$ recorded at three different temperatures (same conditions as in Fig. 8) concerning the first-order optical phonon scattering by the Si substrate. The inset shows how the temperature of the illuminated volume is deduced from the shift of this mode, following the calibration (red line) deduced from the work of Balkanski et al. [38].

the spectrometer response. This response is normalized by taking $f\left(\omega_{i}, 0\right)=1$, where $\omega_{i}$ corresponds to the laser line $\left(\lambda_{i}=413 \mathrm{~nm}\right)$. In the shaded area of Fig. 3(b), the frequency shift $\omega$ varies from 500 to $2000 \mathrm{~cm}^{-1}$. In this spectral range, the response consists in a monotonous curve that will not affect significantly the Raman bands.

The presence of a common Si substrate in all the samples has offered the opportunity to use it as an in situ Raman reference for frequency and temperature calibration. On one hand, checking the low-frequency range on both sides of the Rayleigh line was performed by recording the Si Brillouin signal. On the other hand, heating or cooling of the samples was controlled using the anti-Stokes versus Stokes ratio, the shift and broadening of the first-order lines corresponding to the Raman signal of the Si optical phonon. An example of temperature measurement is reported in Fig. 4: it has been used to determine the local temperature of the sample under investigation (See Fig. 7).

\section{Preparation and characterization of the samples}

The Ag-NCs embedded in dielectrics have been obtained by implantation of $\mathrm{Ag}^{+}$ions with low kinetic energy in thin layers by means of a Varian 200A2 implanter. According to SRIM simulations [39], the energy has been adapted for each layer to get an implanted profile reaching its maximum at a depth $\approx 10 \mathrm{~nm}$ with a straggle of few $\mathrm{nm}$. According to previous work [40], the fluence value was adapted in the range of few $10^{15}$ to few $10^{16}$ ions $/ \mathrm{cm}^{2}$ to obtain assemblies of Ag-NCs with sizes in the nm range with limited dispersion. These NCs result from nucleation and growth processes that occurred during implantation of $\mathrm{Ag}^{+}$ions [37]. A part of the free surface has been masked during implantation to get a reference signal in order to better discriminate the elastic or 


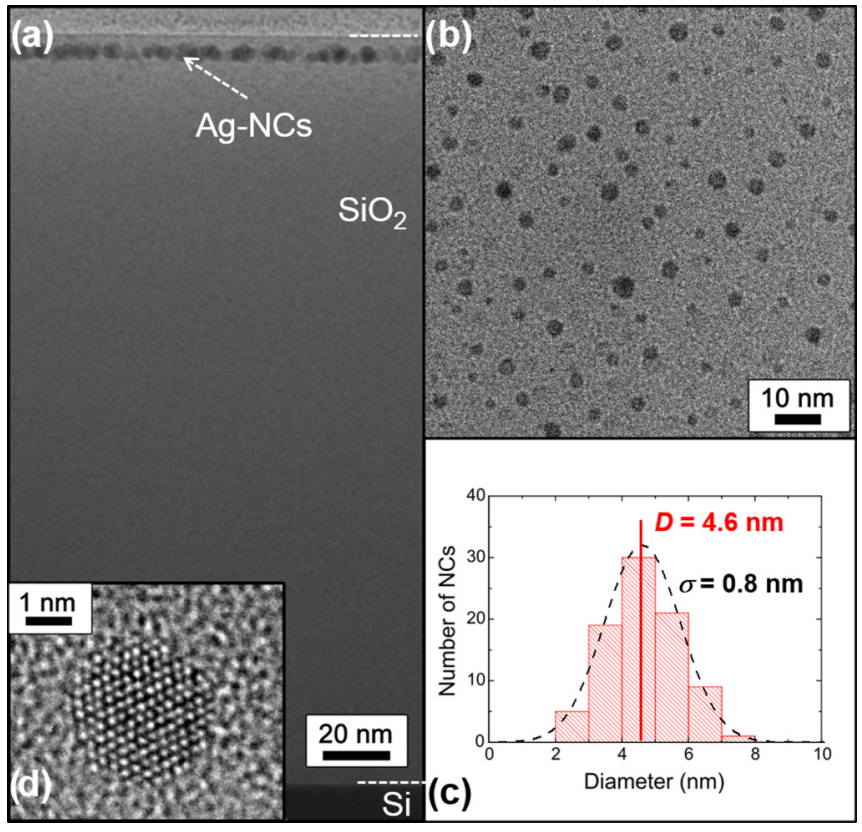

FIG. 5. (Color online) (a) Cross-section TEM image at low magnification of an $\mathrm{SiO}_{2} / \mathrm{Si}$ layer implanted with $\mathrm{Ag}^{+}$ions (sample B); (b) associated plan-view image and (c) associated size distribution (the dotted line corresponds to a fit by a Gaussian with a mean value $D$ and a standard deviation $\sigma$ ); (d) HREM analysis showing one isolated silver nanocrystal.

inelastic signal originating in the NCs. One sample (sample A) was exceptionally implanted at higher energy and dose to get a higher signal for temperature-dependent measurements.

Transmission electron microscopy (TEM) imaging was performed using a field emission FEI Tecnai F20 microscope operating at $200 \mathrm{kV}$. It was equipped with a spherical aberration corrector dedicated for high-quality high-resolution electron microscopy (HREM) images with an increased signal-to-noise ratio and nearly no delocalization effect at surfaces and interfaces. In the case of $\mathrm{Ag}-\mathrm{NCs}$ in $\mathrm{TiO}_{2}$ layer, additional energy-filtered TEM (EFTEM) imaging has been conducted with a TRIDIEM Gatan imaging filter attached to the microscope and coupled to a scanning stage for the STEM-EELS analyses. TEM lamellae transparent to electrons were prepared in cross section and plan view by mechanical grinding and $\mathrm{Ar}^{+}$ion milling. An example of TEM and HREM analysis is reported in Fig. 5.

\section{RESULTS}

\section{A. Stokes and anti-Stokes electronic Raman scattering}

The Raman spectrum recorded at room temperature from a large assembly of Ag-NCs embedded in a thick $\mathrm{SiO}_{2}$ layer (sample A) is reported in Fig. 6 (black line). The spectrum has been recorded in plasmon-resonant conditions using the 413-nm (3.00-eV) line of a krypton laser. TEM measurements have shown that the particles are well isolated from each other, crystalline, and spherical with an average diameter $D \approx 6 \mathrm{~nm}$. Note that the very low-frequency (Raman-Brillouin) range, down to a $4-\mathrm{cm}^{-1}$ shift from the Rayleigh line, has been also recorded (Fig. 2 and inset in Fig. 6).

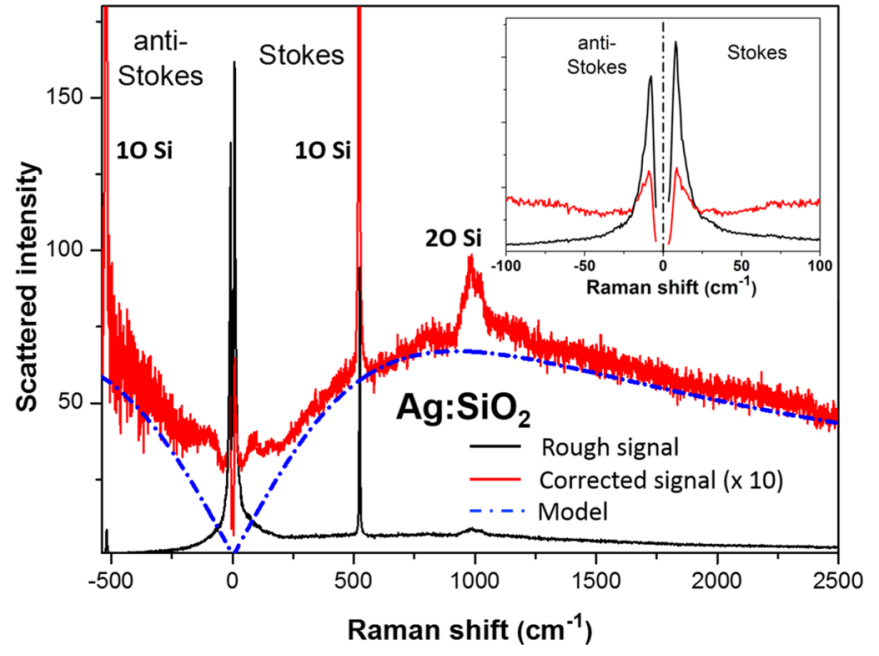

FIG. 6. (Color online) Raman spectrum recorded at room temperature on $\mathrm{SiO}_{2}$ layer containing Ag-NCs (sample A). The exciting wavelength is $413 \mathrm{~nm}$. The dark line corresponds to the rough recorded spectrum. The red line corresponds to the spectrum corrected from the Bose statistical factor, $\omega^{4}$ scattering law, spectral response of the setup, and dark noise. The blue dashed-dotted line results from modeling [Eq. (14)]. The inset displays the low-frequency region.

The rough signal $I^{\text {expt }}$ has been corrected from the Bose statistical population factor $\bar{n}$ and the spectral response $f$ of the whole experimental setup. One thus defines a corrected intensity $I(\omega)$ as follows:

$$
I(\omega)=I(\omega)^{\mathrm{corr}}=I^{\operatorname{expt}}(\omega)\left[|\bar{n}(\omega, T)+1| \times f\left(\omega_{i}, \omega\right)\right]^{-1}
$$

with

$$
\bar{n}=\left[\exp \left(\frac{\hbar \omega}{k_{B} T}\right)-1\right]^{-1} .
$$

In these expressions which are valid for both Stokes and antiStokes parts, $\omega$ is the relative Raman frequency shift $(\omega>0$ for Stokes, and $\omega<0$ for anti-Stokes events) and $\omega_{i}$ is the incident laser frequency [41]. In what follows, all these frequencies are expressed in $\mathrm{cm}^{-1}$ units, as usually done in vibrational spectroscopy.

The corrected signal of sample A is reported in Fig. 6 and in the inset (red lines) with an amplification factor of 10 with regard to the rough signal. One observes that despite fluctuations generated in the anti-Stokes region where $|\bar{n}(\omega, T)+1|$ vanishes for $\hbar|\omega|>k_{B} T$, the symmetry of the response is well verified. As already recently discussed [19], this signal can be decomposed in three components: (i) the low-frequency range (below $40 \mathrm{~cm}^{-1}$ ) dominated by the contribution of quasi-confined elastic waves in Ag-NCs, referred as Lamb modes [42], (ii) the high-frequency range (beyond $200 \mathrm{~cm}^{-1}$ ) where the two Raman peaks corresponding to optical phonons of the $\mathrm{Si}$ substrate (first order $1 \mathrm{O}$ and second order 2O) emerge from a very broad band, the so-called "background," which culminates around $800 \mathrm{~cm}^{-1}$ but expands far beyond this value, and (iii) a less intense intermediate range (between 40 and $200 \mathrm{~cm}^{-1}$ ) attributed to Raman scattering by "bulk phonons" and reflecting the vibrational density of states (VDOS) of the embedded Ag-NCs [19]. 


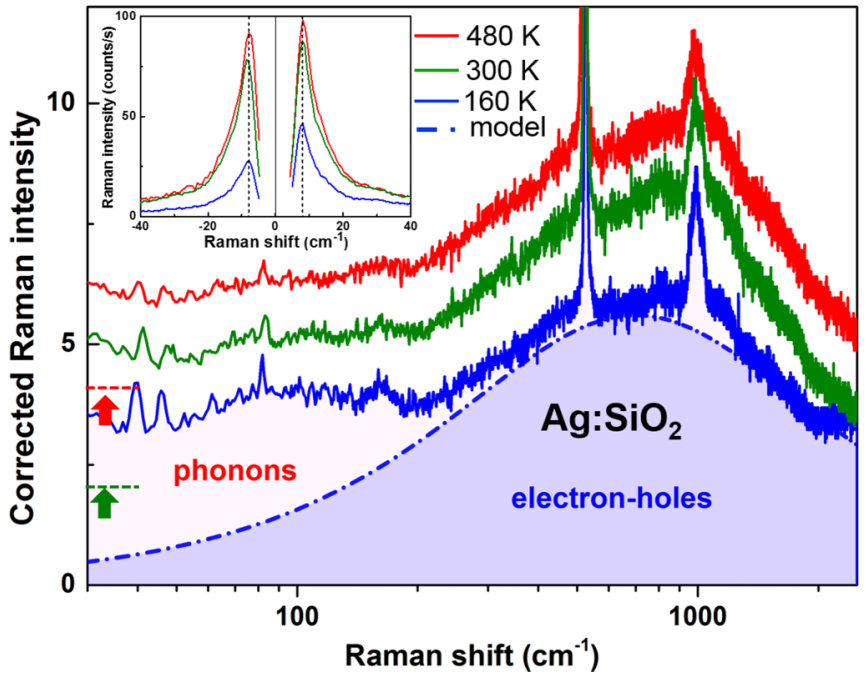

FIG. 7. (Color online) Corrected Raman spectra recorded within the same experimental conditions as in Fig. 6, but at three different temperatures. Note the log scale for the Raman shift. The two spectra recorded at the highest temperatures are arbitrary up-shifted (see arrows) for clarity. The blue dashed-dotted line results from modeling [Eq. (14)]. The low-frequency range, with rough experimental intensity and linear frequency scale, is reported in the inset.

All these three components must be assigned to inelastic scattering events. In particular, the background follows Bose statistics and that comforts unambiguously its interpretation as inelastic scattering by electron-hole pair excitations $[14,17,19,20,22,43]$. In particular, Portales et al. have exactly drawn the same conclusions by analyzing Raman scattering by Ag-NCs embedded in $\mathrm{SiO}_{2}$ but elaborated within a very different technique [17]. They have shown that the signal shape remains the same when changing the excitation wavelength, thus excluding once more a photoluminescence origin.

\section{B. Temperature effect}

We have checked the thermodynamics balance equation for electronic excitations by changing the temperature. The result is displayed in Fig. 7 where the corrected intensity for the same assembly of $\mathrm{Ag}-\mathrm{NCs}$ in $\mathrm{SiO}_{2}$ is reported at three different temperatures. In Eq. (7), the temperature value of the explored volume has been checked using the first-order Raman signal of the Si substrate as a built-in thermometer (see Fig. 4). The corrected intensity is quite unaffected by the temperature modification. This is particularly well verified in the high-frequency range (ii), where only electronic scattering can be concerned: the signal is unchanged even if the thermal energy is tripled, from $k_{B} T=13 \mathrm{meV}$ at $160 \mathrm{~K}$ to $42 \mathrm{meV}$ at $480 \mathrm{~K}$. That rules out once more the implication of photoluminescence processes in this range. One notes that the low-frequency range below $200 \mathrm{~cm}^{-1}$, (i) and (iii), is slightly affected by the temperature change. This dependence is attributed to electron-phonon processes implying bulk phonons whose associated Debye temperature is around $200 \mathrm{~K}$ in nm-sized Ag-NCs [19], i.e., inside the temperature range explored. The Lamb modes spectra (iii) at very low frequency are presented in the inset of Fig. 7 (rough spectra with linear frequency scale). The frequency of these modes is not affected by the temperature change and only their intensity is modified due to thermal population effects.

\section{Size effect}

The average diameter $D$ of an assembly of Ag-NCs can be evaluated by TEM analysis or low-frequency Raman spectroscopy (see Table I). In this work, we choose to deduce the mean diameter of each Ag-NCs assembly from the frequency of the Lamb mode that dominates the low-frequency Stokes and anti-Stokes Raman spectra. Indeed, this in situ signal originates from the total but only volume in which inelastic electronic scattering occurs. Both vibrational and electronic signals should suffer the same confinement and plasmon-resonant enhancement that critically depend on size distribution and excitation energy [44].

The quasiconfined elastic Lamb modes correspond to global deformations of the NC, with corresponding wavelength $\lambda \sim$ $D$. Due to the linear dispersion law of acoustic waves, their frequency varies as $D^{-1}$ :

$$
v_{n, \ell}=A_{n, \ell} \frac{v_{T}}{D}
$$

where $v_{T}$ is the transverse sound velocity and $A_{n, \ell}$ a numerical coefficient. This coefficient depends on the principal and azimuthal quantum numbers $(n, \ell)$ related to spherical symmetry and on the acoustic impedance mismatch between the NC and its surrounding medium. In Fig. 2, one can distinguish three Lamb modes on the Raman spectrum of sample A. The lowest-frequency and highest-intensity mode is ascribed to the fundamental $(n=1)$ quadrupolar $(\ell=2)$ mode. For a spherical Ag-NC embedded in $\mathrm{SiO}_{2}$, it has been shown theoretically [42] and well verified experimentally [34,42] that $A_{1,2}=0.85$. Using the resulting relationship $\omega_{1,2}\left(\mathrm{~cm}^{-1}\right)=$ $47 / D(\mathrm{~nm})$, one deduced an average diameter of $6.0 \pm 0.4 \mathrm{~nm}$ for sample A from its spectrum reported in Fig. 2, in good agreement with TEM measurements (see Table I), but with a lower inhomogeneous broadening, probably due to selective plasmon-resonance effects [44].

For analyzing the size effect in smaller NCs, we used the ion-beam synthesis method but at very low energy $(3 \mathrm{keV})$ to generate the formation of a single plane of small embedded Ag-NCs. This plane is located at the immediate vicinity of the free surface of $\mathrm{a} \mathrm{SiO}_{2}$ layer thermally grown on a Si substrate [34,37]. For a low-ion dose, the NCs are spherical, well separated, and they present a rather narrow size distribution [40]. Here, only three samples with the lowest size dispersion (in the 2-6 nm range) were chosen. Their corrected Raman spectra are reported in Fig. 8 and displayed using a log scale for the frequency shift. This unusual procedure allows a better comparison for the three frequency ranges already discussed.

The intermediate range (iii) is rather unaffected by the size change. It has been recently shown that the corresponding Raman response gives an image of the VDOS of the AgNCs [19]. Van Hove singularities associated to transverse and longitudinal acoustic modes (TA and LA) at the Brillouin zone edges (near the $X$ and $L$ points) are clearly observed (see dotted lines in Fig. 8). Their frequencies are unaffected by size 


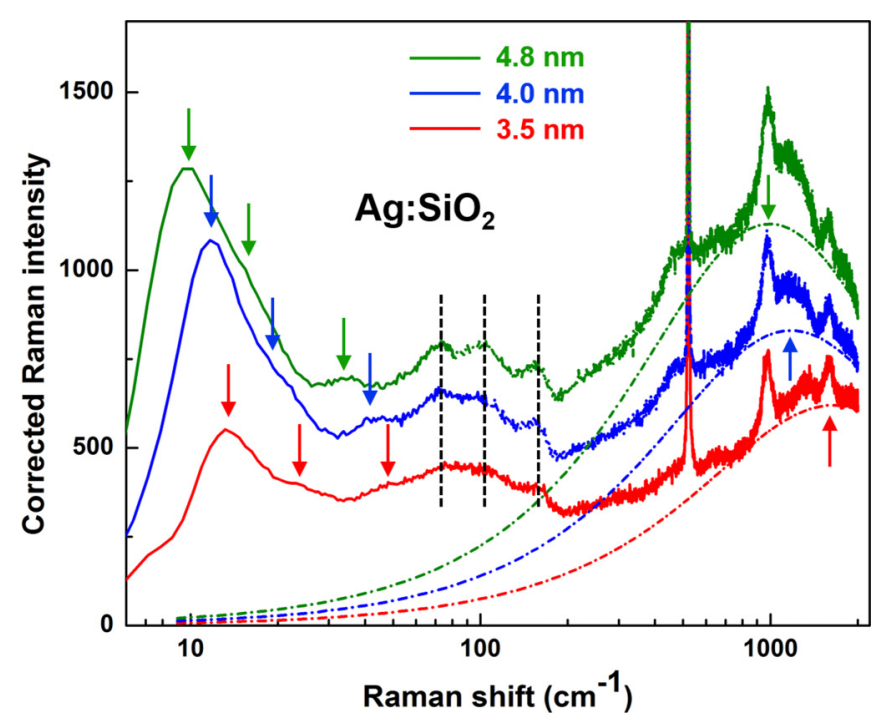

FIG. 8. (Color online) Corrected Raman spectra of three silica layers containing $\mathrm{Ag}-\mathrm{NCs}$ with different average size. The vertical dotted lines are only a guide to show modes that do not depend on size ("bulk phonons"). The various arrows show the size-dependent modes (vibrational Lamb's modes at low frequency, maximum of the electron-hole continuum at high frequency). Note the unusual $\log$ scale for the Raman shift. The dashed-dotted lines result from modeling [Eq. (14)].

change because of their corresponding wavelength remaining at the atomic scale: $\lambda \sim a \ll D$.

On the contrary, the low- (i) and high- (ii) frequency ranges are shifted when the NC sizes are modified, demonstrating their susceptibility to size change. Moreover, whatever the sample, the frequency spacing between their respective maxima remains constant in log scale demonstrating that they follow the same size dependence.

Electronic excitations and acoustic vibrations are both highly confined in these metallic nm-sized systems (diameter $3 \leqslant D \leqslant 6 \mathrm{~nm}$ ). Their corrected signature displays the same geometrical dependence and is not affected by temperature change. One can conclude from this analysis that the frequency maximum of the electronic inelastic scattering band only depends on geometry and displays a linear dependence on the inverse of the average diameter in spherical NCs. The same trend has been already observed by Portales et al. [17].

\section{Matrix effect}

Since confinement plays the prominent role, we have analyzed the role of the embedding matrix. For that purpose, we have elaborated assemblies of Ag-NCs in $\mathrm{Si}_{3} \mathrm{~N}_{4}$ (sample E) and $\mathrm{TiO}_{2}$ (sample $\mathrm{F}$ ), using the same implantation technique. The Raman spectra recorded in plasmon-resonant and optically enhanced conditions in $\mathrm{Si}_{3} \mathrm{~N}_{4}$ and $\mathrm{TiO}_{2}$ are displayed in Figs. 9 and 10, respectively. In both cases, the background still displays the characteristics already assigned to a pure electronic inelastic scattering: symmetry of the Stokes-anti-Stokes corrected spectra, quasilinear frequency dependence at low frequency, and a smoothly decreasing dependence at high frequency. For $\mathrm{Si}_{3} \mathrm{~N}_{4}$, we generally found in TEM measurements a larger size distribution than for $\mathrm{SiO}_{2}$

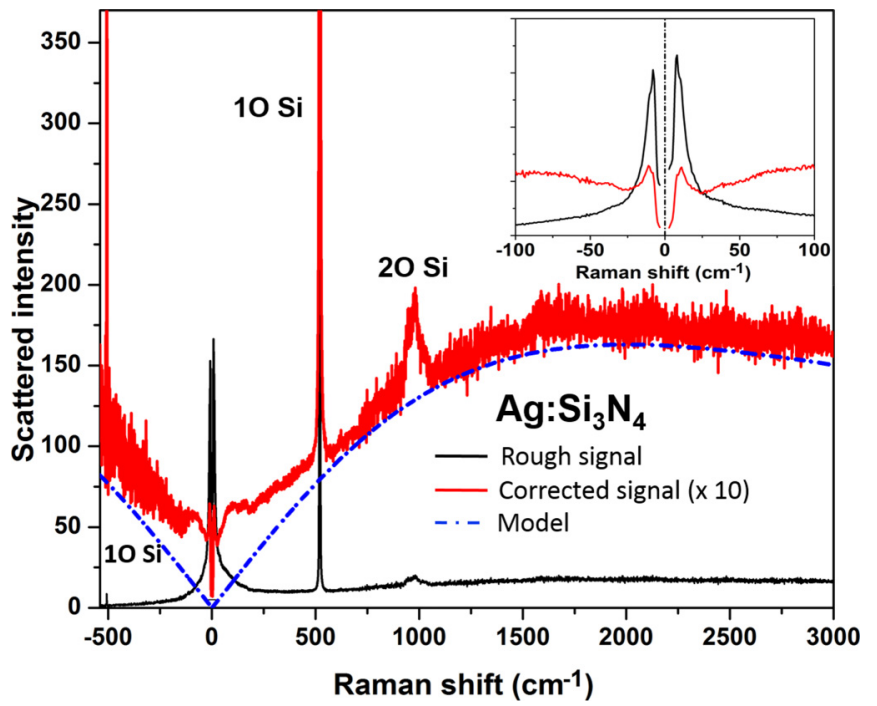

FIG. 9. (Color online) Raman spectra recorded at room temperature on $\mathrm{Ag}-\mathrm{NCs}$ embedded in a $\mathrm{Si}_{3} \mathrm{~N}_{4}$ layer (sample E); the dark line corresponds to the rough recorded spectrum, the red line to the corrected spectrum. The blue dashed-dotted line results from modeling [Eq. (14)]. The inset shows the low-frequency Stokes and anti-Stokes ranges.

samples [45]. The sample used in this work has a size diameter $D=2.8 \pm 0.6$ and this well accounts for the higher value of the background maximum (near $2000 \mathrm{~cm}^{-1}$ ) and the larger broadening observed in Fig. 9. As a matter of fact, the Lamb modes are also more broadened than in $\mathrm{SiO}_{2}$ as can be noted by comparing the insets in Figs 6, 9, and 10.

In the case of $\mathrm{TiO}_{2}$ as embedding medium (Fig. 10), the background signal is more difficult to analyze because aside from the $\mathrm{Si}$ signal, one also observes the signal coming

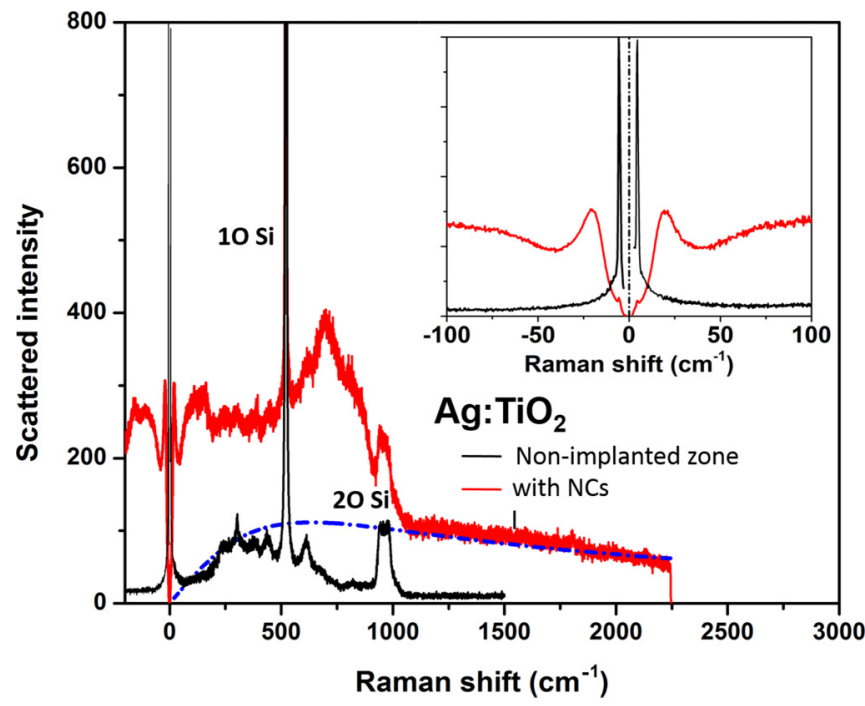

FIG. 10. (Color online) Plasmon-resonant Raman scattering (red line) from Ag-NCs embedded in a $\mathrm{TiO}_{2}$ matrix (sample F). The black curve at bottom shows the reference signal of the $\mathrm{TiO}_{2}$ matrix without NCs. The inset shows the low-frequency Stokes and antiStokes ranges. The blue dashed-dotted line results from modeling [Eq. (14)]. 
from the rutile phase of the polycrystalline $\mathrm{TiO}_{2}$ matrix. In the $600-900 \mathrm{~cm}^{-1}$ frequency range, the recorded signal (red line in Fig. 10) is dominated by SERS by polar longitudinal optical phonons coupled with charged injected carriers [46]. As a matter of fact, the signal recorded within the same experimental conditions on a zone of the sample masked during the $\mathrm{Ag}^{+}$implantation process (black line in Fig. 10) is only composed of Raman-allowed phonons of the Si substrate and crystalline rutile layer. One thus concludes that SERS implies vibrational modes, here Lamb and bulk modes of the Ag-NCs, but also modes of the matrix at the immediate vicinity of these NCs. However, only the electronic contribution remains beyond the VDOS cutoff, i.e., $830 \mathrm{~cm}^{-1}$ for $\mathrm{TiO}_{2}$ [47] and can thus be clearly evidenced.

\section{E. Plasmon-resonant Raman scattering versus hot luminescence}

When the SERS substrate is covered with organic molecules displaying high-frequency vibrational features, the background and these features are mixed and this has led to various interpretations on the signal origin. Moreover, when the metal is gold (or copper) instead of silver, real electronic interband transitions between the $d$ and $s p$ bands are implied in the Raman mechanisms, leading to interference between resonant Raman processes and hot luminescence ones. We have examined these two points by recording Raman spectra from assemblies of Au-NCs functionalized with tris(4,6-dimethyl3-sulfonatophenyl) phosphine trisodium salt hydrate (TDSP), presenting an average size of $5.0 \mathrm{~nm}$. These assemblies were deposited on an antireflective substrate [Fig. 2(b)] and excited with two different laser lines with regard to the LSPR. The corresponding spectra are reported in Fig. 11. On all these spectra one observes inelastic scattering by electronic excitations ("background") on which is superimposed inelastic scattering by vibrational excitations of both Au-NCs [Lamb modes and bulklike phonons: see the inset in Fig. 11(a)] and ligands. When the laser excitation is tuned from $\lambda_{i}=532$ to $638 \mathrm{~nm}$,

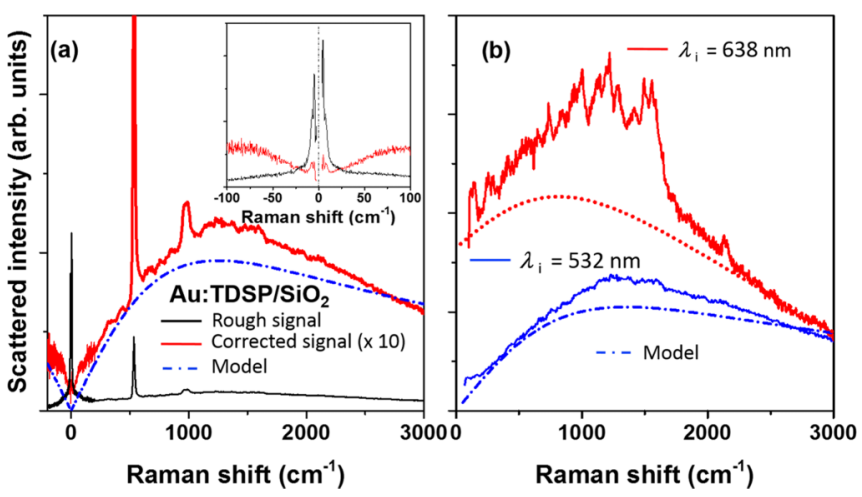

FIG. 11. (Color online) Raman spectra recorded on an assembly of ligand-capped $\mathrm{Au}-\mathrm{NCs}$ deposited on a $\mathrm{SiO}_{2} / \mathrm{Si}$ substrate (sample $\mathrm{G})$ : (a) rough (black line) and corrected spectrum (red line) near plasmon resonance (the inset shows the low-frequency signal); (b) corrected spectra near plasmon resonance (continuous blue line at bottom) and in resonance conditions (638 $\mathrm{nm}$, red line); the signal from the Si substrate has been subtracted. The blue dashed-dotted lines in (a) and (b) result from modeling [Eq. (14)]. The red dotted line in (b) is simply a guide for the eye. near the maximum of the LSPR wavelength $\left(\lambda_{\mathrm{LSPR}} \simeq 630 \mathrm{~nm}\right.$ [20]), one notes in Fig. 11(b) that the response of all excitations displays a similar enhancement, but that the corrected intensity of the inelastic signal does not goes to zero at zero-frequency shift. A similar behavior for the background and molecular signals, as already noted $[27,29,48]$, may suggest that similar resonant Raman scattering processes have to be considered for vibrations and electron-hole pairs. However, the finite value of the corrected intensity at low frequency, near the elastic line, cannot be attributed to Raman processes but to the occurrence of hot luminescence $[49,50]$. This phenomenon has never been observed with clean Ag-NCs, and silver is well known, and obviously largely used, for the absence of any electronic direct interband transition in the visible range. On the contrary, interband single electron-hole excitations from occupied $d$ states to free $s p$ states above the Fermi level, have to be taken into consideration in gold: they occur below $730 \mathrm{~nm}$ near the $X$ point of the Brillouin zone, and are important around $520 \mathrm{~nm}$ near the $L$ point $[49,51]$. Moreover, the presence of the TDSP capping molecules does alter the NCs electronic wave function at the vicinity of the metal surface making really complex any interpretation of the shape of this "hot" background [21,22]. As a consequence, controversial interpretations of the background signal still remain, in particular with gold-based plasmonics structures operating in the visible range, as already underlined recently $[22,50]$.

To go further in the theoretical interpretation and fit of electronic inelastic scattering by $M-\mathrm{NCs}$, one thus has to resume its main characteristics. First, this so-called background signal is always present in nm-sized noble metal structures and absent on free flat surfaces. However, in the latter case, it can be induced by the presence of a molecule, a metallic tip, or any structure creating an electronic contact, an electronic or optical gap at the nanoscale. Its activation is thus always linked to the lack of translational invariance of the metallic surface and its spectral shape is governed by an associated spatial "size" $D$. Second, at thermal and electronic equilibrium, the spectral and temperature background dependence follows Bose statistics as any inelastic scattering process, as a consequence of the principle of detailed balance in thermodynamics. Third, if any absorption or emission process from real direct single electron-hole transitions can be excluded, the background shape of the signal presents a universal shape with an affine linear dependence at low frequency, a broad maximum and a slowly decreasing tail at high frequency [Figs 6, 7, 8, 9, and 11(a)].

\section{DISCUSSION}

\section{A. Raman scattering cross section}

To account for these results, one has first to come back to considerations regarding inelastic light scattering by a continuum of vibrational or electronic excitations. For a long time Raman scattering spectroscopy was predominantly devoted to the study of vibrations in molecules or phonons in ordered crystals whose signatures are narrow (few $\mathrm{cm}^{-1}$ ) and often intense lines. So, these signatures have been used as sensitive probes for analyzing atomic arrangements, strain, doping, temperature, applied fields .... On the contrary, for 
vibrations in highly disordered materials (amorphouslike) or similarly for free electrons in metals, the Raman scattering response consists in a continuous signal smeared over a wide range of frequencies (few hundreds or thousands of $\mathrm{cm}^{-1}$ ) and it is several orders of magnitude lower [30,41]. Experimental studies on these systems are thus less numerous and this type of signal is often discarded as a "noise" or sometimes assimilated to a luminescence "background" like in SERS spectra. Moreover, a quantitative interpretation of the spectrum preliminary needs accurate corrections for temperature effects and setup response.

Whatever the nature of the excitation involved, mechanical or electronic, the Stokes (or anti-Stokes) Raman scattering rate, from an initial state $|i\rangle$ to a final one $|f\rangle$ containing one extra (or missing, respectively) excitation, can be written as [52]

$$
\begin{aligned}
\mathcal{P}\left(\omega_{i}, \omega\right)= & \frac{2 \pi}{\hbar} \mid \sum_{a, b} \frac{\left\langle f\left|H_{v-e}\right| b\right\rangle\left\langle a\left|H_{v-e}\right| i\right\rangle}{\left(\hbar \omega_{s}-E_{b}-i \Gamma_{b}\right)\left(\hbar \omega_{i}-E_{a}-i \Gamma_{a}\right)} \\
& \times\left.\left\langle b\left|H_{e-e x}\right| a\right\rangle\right|^{2} \delta\left(\omega_{i}-\omega_{s}-\omega\right) .
\end{aligned}
$$

In this expression, the summation is performed over intermediate states $|a\rangle$ and $|b\rangle$ between which the interaction $H_{e-e x}$ between the photoexcited electron and the elementary excitation (electron-hole or vibration) takes place. The broadening of the electronic states with energy $E_{a}$ or $E_{b}$ is described by introducing phenomenological parameters $\Gamma_{a}$ or $\Gamma_{b}$. From general considerations on time-dependent perturbations in quantum mechanics, it is known that all the orders of the different steps reported in Eq. (9) have to be considered. We have formally isolated the matrix element $\left\langle b\left|H_{e-e x}\right| a\right\rangle$ from the two others relative to incident $\left\langle a\left|H_{v-e}\right| i\right\rangle$ and scattered $\left\langle f\left|H_{v-e}\right| b\right\rangle$ photon-electron (or more exactly polariton-plasmon) interaction. These latter terms account for SERS mechanisms, either electromagnetic (EM) or chemical (CM) one. The enhancement associated to LSPR is accounted for by the associated terms in the denominator. As they are independent from the type of excitation involved, the scattering rate for either vibrational or electronic excitations is expected to follow rather the same enhancement behavior versus the incident and scattered energy $\left(\hbar \omega_{i}\right.$ and $\left.\hbar \omega_{s}\right)$. Their respective frequency line shape and selection rules are mainly accounted for by the $H_{e-e x}$ matrix elements.

Whereas energy has only to be globally conserved, symmetry selection rules must be satisfied at each step of the process, in particular for the $H_{e-e x}$ interaction step. In a crystal, the momentum must be conserved and only direct transitions $\left(\mathbf{q}=\mathbf{k}_{i}-\mathbf{k}_{s} \simeq \mathbf{0}\right)$ are allowed because the light momentum $q$ transferred in backscattering geometry is of the order of $0.02 \mathrm{~nm}^{-1}$ in the visible range $(\lambda \approx 600 \mathrm{~nm})$, and thus negligible in comparison with the Brillouin zone size $(\approx$ $10 \mathrm{~nm}^{-1}$ ). An elementary electronic Raman scattering process in a metal consists in the excitation of an electron from below to above the Fermi level. In the particular case of an ideal Drudetype gas, electronic Raman scattering is expected to be very low because such intraband excitation is forbidden. It cannot simultaneously satisfy energy and momentum conservation rules due to the great difference between electron and photon dispersion relations. However, due to the finite penetration depth $\delta$ of the light in the visible-UV range in real metals, this restriction is less drastic. In noble metals, such as $\mathrm{Ag}$ where $2 / \delta \approx 0.1 \mathrm{~nm}^{-1}$ and the Fermi surface is isotropic, the activation for inelastic electronic scattering is not very likely and the signal remains very weak. In other elemental metals having anisotropic Fermi surfaces and/or lower penetration depth, the scattering rate can be somewhat higher. Thanks to the development of wide aperture collecting systems and efficient CCD detectors, scarce measurements of Raman scattering by electrons in elemental metals have been recently performed [32]. One has to mention that, on the contrary, numerous studies have concerned high- $T_{c}$ superconductors where electronic Raman scattering is highly activated via strong electron-phonon interactions [53]. In such materials, or more generally in highly correlated systems, inelastic light scattering by free electrons has provided precious insights into many-body behavior and electron dynamics near the Fermi surface [31].

\section{B. Spectral response for electronic scattering}

Coming back to normal metals, but containing impurities, a new scaling length is defined by the associated mean-free path for electrons $\bar{d}$. When the level of impurity is high, $1 / \bar{d} \gg q$, allowing to overcome the momentum rule barriers. Hence, the scattering rate for inelastic electronic scattering becomes appreciable. Theoretical approaches have been developed to account for Raman scattering in such metals within a so-called "dirty-metal" model [30]. In the dirty limit, defects, impurities, phonons, or other strong interactions reduce the carriers mean-free path of carriers. In that case it has been shown how inelastic electron scattering is determined by electron population at the vicinity of the Fermi level. In isotropic and noninteracting electron gas, the collision-limited electronic susceptibility is described by a function directly linked to the associated Drude-type conductivity [30,31].

Leaving resonance phenomena aside, the cross section for Raman scattering by intraband excitations for both Stokes and anti-Stokes processes is then written as

$$
I^{\operatorname{expt}} \propto|\bar{n}(\omega, T)+1| N_{F} \frac{|\omega| \tau_{s}}{1+\left(\omega \tau_{s}\right)^{2}},
$$

where $N_{F}$ is the density of states at the Fermi level and $\tau_{s}=$ $\bar{d} / v_{F}$ an "effective" collision time. A similar expression has been shown to also account for electronic Raman scattering in the normal state of conventional elemental metals [32] and in highly doped semiconductors [33]. It is known that the Raman response is linked to the imaginary part $\chi^{\prime \prime}(\omega)$ of the dielectric susceptibility of the system. For free electrons, it has been demonstrated that there is a simple correspondence between the real part of the conductivity $\sigma^{\prime}(\omega)$ and $\chi^{\prime \prime}(\omega)$ following [31]:

$$
\chi^{\prime \prime}(\omega) \propto \omega \sigma^{\prime}(\omega)
$$

highlighting that electronic Raman scattering measures transport properties. Using a simple isotropic Drude model for conductivity, that is relevant in a noble metal, one gets

$$
\sigma(\omega)=\frac{\sigma_{0}}{1-i \omega \tau}
$$


One deduces the following expression for the corrected intensity:

$$
I(\omega) \propto \chi^{\prime \prime}(\omega) \propto \frac{\sigma_{0}|\omega|}{1+(\omega \tau)^{2}}
$$

which coincides with Eq. (10).

Using the same semiclassical approach, it is obvious to consider that in a metallic particle of size $D$ of few $\mathrm{nm}$, the "effective" electron mean-free path is reduced to $\approx D$ by surface collisions, as for Landau damping phenomenon [see Eq. (4)] [36]. The mean-free path in bulk Ag or Au is indeed higher than $D$ because $\bar{d} \approx 40 \mathrm{~nm}$ at room temperature. In the quantum description of the scattering rate [Eq. (9)], a correspondence is given by the fact that, due to electronic confinement in a system of size $D$, the wave-vector selection rule is relaxed in the range $\Delta k \approx 2 \pi / D$ for calculating the $\left\langle b\left|H_{\text {e-ex }}\right| a\right\rangle$ transition matrix elements. For $D=4 \mathrm{~nm}$, one gets $q \ll 1 / D$ as in a "dirty" limit. Here, the mean-free path is not determined by the impurity amount like in doped semiconductors [33], or by the electron-phonon interaction strength like in superconductors $[30,31]$, but by the size of the NCs. The signal will thus be composed of a continuum "background" spread over a frequency range increasing as $1 / D$ and will obviously not be affected by a temperature change, as observed experimentally. In Eq. (9), the finite-size effect affects all the steps, namely, also the light-matter interactions $\left(H_{v-e}\right)$ because $D$ determines the plasmon-polariton field localization. This accounts for the broadening of the LSPR, following Eq. (4) and its similar $1 / D$ dependence.

The theoretical spectra defined by Eq. (10) is reported in Fig. 12 for three values of the temperature and using an arbitrary value for $1 / \tau_{s}=600 \mathrm{~cm}^{-1}$ in wave-number units, i.e., $\Gamma_{s}=\hbar / \tau_{s}=75 \mathrm{meV}$ in corresponding energy units. This figure shows evidence that these spectra do not go to zero at low-frequency limit and present a tail extending in

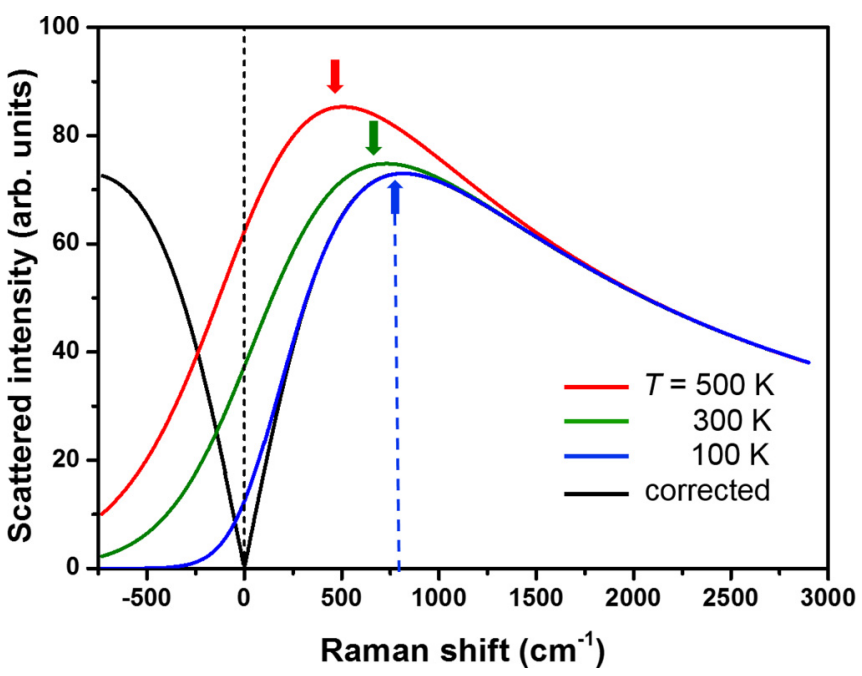

FIG. 12. (Color online) Theoretical Raman scattering cross section for electronic inelastic scattering [Eq. (10)] in metallic nanostructures for three different temperatures. The corrected spectrum (in black) takes into account the Bose statistics population factor [Eq. (7)]. The dotted line refers to the value of the parameter $1 / \tau_{s}$ $\left(600 \mathrm{~cm}^{-1}\right.$ in wave-number units). a continuous way to the anti-Stokes region. The shape and position of the maximum of the Raman band (see arrows in Fig. 12) are temperature dependent and the anti-Stokes signal rapidly increases upon temperature. This may particularly occur when some intense and/or pulsed laser excitation generates a high-temperature electron gas [54]; however, the signal has still to be interpreted as inelastic light scattering by free carriers [50]. This can even be useful to determine the free-electron gas temperature that can considerably differ from the lattice temperature (deducible for instance from Raman signatures of lattice vibrations). At thermal equilibrium, one can extract a temperature-independent line shape, using Eqs. (4), (6), and (10):

$$
I(\omega)=I_{\max } \frac{2|\omega| \tau_{s}}{1+\left(\omega \tau_{s}\right)^{2}} .
$$

This universal expression of the corrected Raman intensity for inelastic electron scattering is reported in Fig. 12 (continuous black line). It can fruitfully be compared with corrected Raman spectra. In particular in the low-frequency range $\left(\hbar|\omega|<k_{B} T\right.$ ), only the corrected signal can give clear insights on the origin of the scattering, analyzing if it vanishes or not. The corrected Raman spectrum is expected to grow linearly at low frequency and then decrease as $1 /|\omega|$ after reaching a maximum $I_{\max }$ when $|\omega|=\Gamma_{s} / \hbar=1 / \tau_{s}$. This is indeed what is observed experimentally and thus the corrected spectra have been fitted using Eq. (14) with $\Gamma_{s}$ as the only adjustable parameter. The agreement with the model is excellent as shown in Figs 6, 7, 8, 9, and 11(a). Our modeling of the electron-hole spectra contrasts with all previous attempts that do not consider the whole frequency range [5,21].

\section{Determination of $g_{s}$ parameter}

The experimental values of $\Gamma_{s}$ for $\mathrm{Ag}-\mathrm{NCs}$ embedded in $\mathrm{SiO}_{2}$ are reported in Fig. 13 (full squares) as a function of the inverse of the mean diameter $D^{-1}$. On this figure are also reported (empty squares) values deduced from already published Raman spectra of Ag clusters embedded in $\mathrm{SiO}_{2}$ [17]. One verifies that our model not only well accounts for the main characteristic of the "background" shape, but also for the linear dependence of its maximum frequency versus the confinement parameter $D^{-1}$, following Eqs. (15) and (14).

The confinement similarly affects both acoustic and electronic waves, following a $D^{-1}$ behavior, but their dynamical scales are very different, in the femtosecond or picosecond regime, respectively. This is well understood by calculating the ratio between $\Gamma_{s}$ and the energy of the dominant Lamb mode, that is size independent:

$$
\frac{\Gamma_{s}}{h v_{1,2}}=\frac{g_{s}}{\pi A_{1,2}} \frac{v_{F}}{v_{T}} .
$$

Since the parameters $g_{s}$ and $A_{1,2}$ are of the order of unity, this ratio is mainly determined by that of the Fermi velocity versus the transverse sound velocity, i.e., $\approx 840$ in bulk silver. In practice, this allows to easily discriminate between vibrational and electronic SERS contributions in noble metal nanostructures, referred as (i) and (ii) previously. On the contrary, when optical phonons of an embedding medium or modes of capping molecules are present, their signatures 


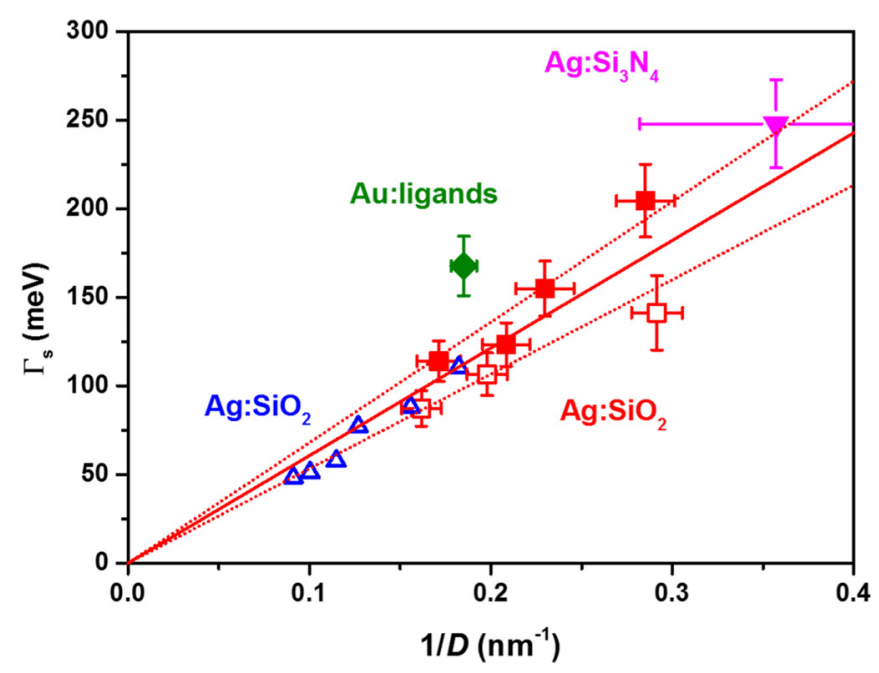

FIG. 13. (Color online) Surface-plasmon damping parameter $\Gamma_{s}$ versus the reciprocal average diameter $D^{-1}$ in $M$-NCs. The full squares correspond to the present experimental work. The empty squares (in red) are extracted from the experimental work of Portales et al. [17]. The empty triangles (in blue) are deduced from the theory [from Lermé et al. [55] using Eq. (4)]. The full and dotted lines (in red) correspond to modeling using Eq. (4) with $g_{s}^{\text {expt }}=0.33 \pm 0.04$.

superimpose and may blend with the electronic scattering signal as observed in Figs 10 and 11, rendering any fitting more hazardous.

In silver, luminescence processes from Ag-NCs are highly unlikely because only intraband transitions are expected to occur under optical excitation in the visible range (below $3 \mathrm{eV}$ ) due to the higher value $(3.9 \mathrm{eV})$ of the $d-s p$ interband transition threshold. It has been shown that the dielectric permittivity is well accounted for by using a simple Drude approximation [35]. The LSPR is thus not damped by interband transitions and in spherical nm-sized NCs where radiation damping and multipole effects are negligible, its width $\Gamma$ is given by adding to the bulk contribution $\Gamma_{\infty}$ the size-dependent contribution $\Gamma_{s}$ given by Eq. $4[35,36]$ :

$$
\Gamma=\Gamma_{\infty}+2 \hbar g_{s} \frac{v_{F}}{D} .
$$

This linear relation of the FWHM of the absorption peak versus $D^{-1}$ has been verified experimentally by time-resolved experiments on isolated nanoparticles. A recent work on single silver nanoparticles coated with a silica shell gave $g_{s}=0.7 \pm 0.1$ with $\Gamma_{\infty}=125 \pm 10 \mathrm{meV}$ [56]. On another hand, many theoretical approaches have been developed since the pioneering work of Kawabata and Kubo [36]: using a simple spherical quantum box model they found $g_{s}=\frac{3}{2}$. More sophisticated models have given generally lower but, however, rather spread values: for a review, see Lermé et al. [55]. In this last work, calculations have been performed using the time-dependent local density approximation, and gave for nm-sized Ag-NCs embedded in SiO2: $g_{s}^{\text {theor }}=0.32$ and $\Gamma_{\infty}=104 \mathrm{meV}$. We have also plotted in Fig. 13 (empty triangles) the theoretical values of $\Gamma_{s}=\Gamma-\Gamma_{\infty}$, following Eq. (16) and using the data given in Ref. [55]. They are in good agreement with the experimental data, if one takes into account the experimental uncertainties on the diameter value $D$ due to inhomogeneous broadening, and on $\Gamma_{s}$ due to spectral response correction. By considering these uncertainties (see dotted lines in Fig. 13), one gets $g_{s}^{\text {expt }}=0.33 \pm 0.04$ which is very close to $g_{s}^{\text {theor }}$. This agreement contrasts with all previous experimental determinations that have given too high values (from 0.7 to 1 ). To explain these disagreements, Lermé et al. have invoked a supplementary electron-phonon scattering contribution, leading to a "corrected" experimental value of $g_{s}^{\text {corr }}=0.4 \pm 0.1$ in agreement with theory. One may argue that in our approach, based on a frequency-scaled analysis rather than on a time-scaled analysis, the electronic contribution can better be isolated (see Fig. 7). Finally, in Fig. 13 we have also reported the experimental results for Ag-NCs in $\mathrm{Si}_{3} \mathrm{~N}_{4}$ and Au-NCs with ligands. Obviously, no accurate value of $g_{s}$ can be obtained from these too scarce data. Moreover, one has to keep in mind that inhomogeneous broadening due to size distribution affects the accuracy of the data, particularly in the case of $\mathrm{Ag}-\mathrm{NCs}$ in $\mathrm{Si}_{3} \mathrm{~N}_{4}$ (see Fig. 9). Nevertheless, our data may be indicative of slightly different values of $g_{s}^{\text {expt }}$ demonstrating that this parameter is depending on the metal and the environment through the electronic surface spill-out and confining potential profile, as predicted theoretically [55].

\section{CONCLUSIONS}

In noble metal nanostructures, the interactions between light, electronic, and vibrational excitations are widely modified because the geometrical parameters are significantly smaller than the light wavelength, skin depth, mean-free path, or $\mathrm{THz}$ acoustic wavelength.... The resulting confinement effects on collective electronic and vibrational modes have well explained the occurrence of surface plasmons or Lamb modes, respectively. In this work, we focused more on the confinement of single-particle electronic excitations and we showed that it plays a prominent role to account for inelastic Raman scattering, in a similar way as for plasmon dephasing (Landau mechanism), using the same geometrical parameter $g_{s}$. We thus confirmed that the so-called "background" is an intrinsic phenomenon of SERS and we have well accounted for its activation, spectral line shape, and size dependence by applying a dirty-metal model early developed for describing electronic scattering in high- $T_{c}$ superconductors. In silver nanostructures where intraband transitions are absent in the visible range, and in the absence of grafted molecules, the background is still always present and its behavior versus temperature and Raman frequency shift is well described as resulting from temperature population effects and geometrical confinement. The role of surface curvature that breaks the momentum conservation requirement for any elementary interaction is expressly verified. This well explains how roughness at the nanoscale or any spatial inhomogeneity at this scale (such as lithographical nanogaps, sharp protusions, apex of a tip, grafted molecule) does play a crucial role in SERS effect. Electronic polarizability, like conductivity, is greatly affected by "collisions" in the fs regime and resulting fluctuations give their unavoidable spectral signature in Raman scattering spread over hundreds or thousands of wave numbers.

Further works are needed to improve more deeply our results. On one hand, from the experimental point of view, 
measurements on better controlled size systems (eventually on isolated nano-objects) and time-resolved inelastic scattering would help to get new insights on confinement effects and discriminate between Raman and photoluminescence processes. On the other hand, for theory, it is crucial to go beyond classical approaches or grid-based methods versus "quantum" plasmonics to take plainly into account intrinsic local effects (i.e., quantum electrodynamical effects such as band-structure modification, level discretization, charge tunneling and dephasing, electron spill-out, multiple-order contribution...). It is of prime importance to describe at the local scale electronic excitations at the vicinity of the Fermi level and how a molecule adsorbed on a metallic surface modifies these excitations to fully understand not only electron inelastic scattering, but also the chemical mechanism for SERS, inelastic electron tunneling, or any plasmochemistry or plasmoelectronic effect at the nanoscale.

\section{ACKNOWLEDGMENTS}

This research has been supported by the FP7 European Project WATER (Grant Agreement No. 316082) whose coordination was assumed by V.P., and part of this work was supported by Programme Investissements d'Avenir under the Program No. ANR-11-IDEX-0002-02, Reference No. ANR10-LABX-0037-NEXT. The authors thank B. Pécassou, A. Zwick, M. Zimbone, A. Slaoui, and N. M. Sangeetha for technical assistance.
[1] M. Fleischmann, P. Hendra, and A. McQuillan, Chem. Phys. Lett. 26, 163 (1974).

[2] J. A. Dieringer, A. D. McFarland, N. C. Shah, D. A. Stuart, A. V. Whitney, C. R. Yonzon, M. A. Young, X. Zhang, and R. P. Van Duyne, Faraday Discuss. 132, 9 (2006).

[3] K. Kneipp, Y. Wang, H. Kneipp, L. T. Perelman, I. Itzkan, R. Dasari, and M. Feld, Phys. Rev. Lett. 78, 1667 (1997).

[4] S. Nie, Science 275, 1102 (1997).

[5] B. Pettinger, K. F. Domke, D. Zhang, R. Schuster, and G. Ertl, Phys. Rev. B 76, 113409 (2007).

[6] T. Schmid, L. Opilik, C. Blum, and R. Zenobi, Angew. Chem. 52, 5940 (2013).

[7] N. Mauser and A. Hartschuh, Chem. Soc. Rev. 43, 1248 (2014).

[8] M. Barbry, P. Koval, F. Marchesin, R. Esteban, A. G. Borisov, J. Aizpurua, and D. Sánchez-Portal, Nano Lett. 15, 3410 (2015).

[9] M. Moskovits, Rev. Mod. Phys. 57, 783 (1985).

[10] A. Otto, J. Raman Spectrosc. 36, 497 (2005).

[11] Y. Fang, N.-H. Seong, and D. D. Dlott, Science 321, 388 (2008).

[12] M. Moskovits, Phys. Chem. Chem. Phys. 15, 5301 (2013).

[13] E. Burstein, Y. Chen, C. Chen, S. Lundquist, and E. Tosatti, Solid State Commun. 29, 567 (1979).

[14] A. Otto, J. Timper, J. Billmann, G. Kovacs, and I. Pockrand, Surf. Sci. 92, L55 (1980).

[15] T. Furtak and J. Reyes, Surf. Sci. 93, 351 (1980).

[16] R. Monreal, F. Flores, Y. Gao, and T. López-Ríos, Europhys. Lett. 4, 115 (1987).

[17] H. Portales, E. Duval, L. Saviot, M. Fujii, M. Sumitomo, and S. Hayashi, Phys. Rev. B 63, 233402 (2001).

[18] J. T. Hugall, J. J. Baumberg, and S. Mahajan, J. Phys. Chem. C 116, 6184 (2012).

[19] M. Bayle, P. Benzo, N. Combe, C. Gatel, C. Bonafos, G. Benassayag, and R. Carles, Phys. Rev. B 89, 195402 (2014).

[20] M. Bayle, N. Combe, N. M. Sangeetha, G. Viau, and R. Carles, Nanoscale 6, 9157 (2014).

[21] L. L. T. Ngoc, J. Wiedemair, A. van den Berg, and E. T. Carlen, Opt. Express 23, 5547 (2015).

[22] J. T. Hugall and J. J. Baumberg, Nano Lett. 15, 2600 (2015).

[23] J. Stadler, T. Schmid, and R. Zenobi, Nano Lett. 10, 4514 (2010).

[24] R. Zhang, Y. Zhang, Z. C. Dong, S. Jiang, C. Zhang, L. G. Chen, L. Zhang, Y. Liao, J. Aizpurua, Y. Luo, J. L. Yang, and J. G. Hou, Nature (London) 498, 82 (2013).
[25] S. A. Maier, Plasmonics: Fundamentals and Applications (Springer, Berlin, 2007).

[26] J. Jiang, K. Bosnick, M. Maillard, and L. Brus, J. Phys. Chem. B 107, 9964 (2003).

[27] T. Itoh, V. Biju, M. Ishikawa, Y. Kikkawa, K. Hashimoto, A. Ikehata, and Y. Ozaki, J. Chem. Phys. 124, 134708 (2006).

[28] S. Mahajan, R. M. Cole, J. D. Speed, S. H. Pelfrey, A. E. Russell, P. N. Bartlett, S. M. Barnett, and J. J. Baumberg, J. Phys. Chem. C 114, 7242 (2010).

[29] C. Farcau and S. Astilean, Chem. Commun. (Cambridge, England) 47, 3861 (2011).

[30] A. Zawadowski and M. Cardona, Phys. Rev. B 42, 10732 (1990),

[31] T. P. Devereaux and R. Hackl, Rev. Mod. Phys. 79, 175 (2007).

[32] Y. S. Ponosov and S. V. Streltsov, Phys. Rev. B 86, 045138 (2012).

[33] G. Contreras, A. K. Sood, and M. Cardona, Phys. Rev. B 32, 924 (1985).

[34] R. Carles, C. Farcau, C. Bonafos, G. Benassayag, M. Bayle, P. Benzo, J. Groenen, and A. Zwick, ACS Nano 5, 8774 (2011).

[35] C. F. Bohren and D. R. Huffman, Absorption and Scattering of Light by Small Particles (Wiley, New York, 1983).

[36] A. Kawabata and R. Kubo, J. Phys. Soc. Jpn. 21, 1765 (1966).

[37] R. Carles, C. Farcau, C. Bonafos, G. Benassayag, B. Pécassou, and A. Zwick, Nanotechnology 20, 355305 (2009).

[38] M. Balkanski, R. F. Wallis, and E. Haro, Phys. Rev. B 28, 1928 (1983).

[39] J. Biersack and L. Haggmark, Nucl. Instrum. Methods, Phys. Res. Sect B 174, 257 (1980).

[40] P. Benzo, C. Bonafos, M. Bayle, R. Carles, L. Cattaneo, C. Farcau, G. Benassayag, B. Pecassou, and D. Muller, J. Appl. Phys. 113, 193505 (2013).

[41] A. Zwick and R. Carles, Phys. Rev. B 48, 6024 (1993).

[42] B. Palpant, H. Portales, L. Saviot, J. Lermé, B. Prével, M. Pellarin, E. Duval, A. Perez, and M. Broyer, Phys. Rev. B 60, 17107 (1999).

[43] W. Akemann and A. Otto, Surf. Sci. 307-309, 1071 (1994).

[44] G. Bachelier and A. Mlayah, Phys. Rev. B 69, 205408 (2004).

[45] M. Bayle, C. Bonafos, P. Benzo, G. Benassayag, B. Pécassou, L. Khomenkova, F. Gourbilleau, and R. Carles, Appl. Phys. Lett. 107, 101907 (2015). 
[46] G. Cacciato, M. Bayle, A. Pugliara, C. Bonafos, M. Zimbone, V. Privitera, M. G. Grimaldi, and R. Carles, Nanoscale 7, 13468 (2015).

[47] S. Schöche, T. Hofmann, R. Korlacki, T. E. Tiwald, and M. Schubert, J. Appl. Phys. 113, 164102 (2013).

[48] S. M. Barnett, N. Harris, and J. J. Baumberg, Phys. Chem. Chem. Phys. 16, 6544 (2014).

[49] M. R. Beversluis, A. Bouhelier, and L. Novotny, Phys. Rev. B 68, 115433 (2003).

[50] J. Huang, W. Wang, C. J. Murphy, and D. G. Cahill, Proc. Natl. Acad. Sci. USA 111, 906 (2014).

[51] G. T. Boyd, Z. H. Yu, and Y. R. Shen, Phys. Rev. B 33, 7923 (1986).
[52] A. Pinczuk and E. Burstein, in Light Scattering in Solids I: Introductory Concepts, edited by M. Cardona (Springer, Berlin, 1983), pp. 23-78.

[53] A. Abrikosov and L. Falkovskii, Zh. Eksp. Teor. Fiz. 40, 262 (1961) [Sov. Phys.-JETP 13, 179 (1961)].

[54] M. Kornbluth, A. Nitzan, and T. Seideman, J. Chem. Phys. 138, 174707 (2013)

[55] J. Lermé, H. Baida, C. Bonnet, M. Broyer, E. Cottancin, A. Crut, P. Maioli, N. Del Fatti, F. Vallee, and M. Pellarin, J. Phys. Chem. Lett. 1, 2922 (2010).

[56] H. Baida, P. Billaud, S. Marhaba, D. Christofilos, E. Cottancin, A. Crut, J. Lermé, P. Maioli, M. Pellarin, M. Broyer, N. Del Fatti, F. Vallée, A. Sánchez-Iglesias, I. Pastoriza-Santos, and L. M. Liz-Marzán, Nano Lett. 9, 3463 (2009). 